\section{Comparison of Musculoskeletal Strength and Body Composition of Hong Kong Chinese Rugby Players, Dragon Boat Paddlers and Controls}

Robin R Mellecker ${ }^{1-3}$, Shirley Siu Ming Fong ${ }^{1}$, Duncan James Macfarlane ${ }^{1}$, Joni $\mathrm{H}$ Zhang $^{1}$ and Ka Ming Wu

\begin{abstract}
Objective: This study used a cross-sectional experimental design to assess musculoskeletal strength, body composition and physical activity levels in Hong Kong Chinese dragon boat paddlers, rugby team players and controls.

Methods: Sixty undergraduate male students (rugby players, $\mathrm{n}=20$ dragon boat, $n=20$ and controls, $n=20$ ) were recruited from a local university. A One-way ANCOVA model was designed with bone strength, muscle strength, flexibility and body composition scores as the independent variables and the three groups as dependent variables.

Results: Significant between-group differences in handgrip strength, flexibility, fat free mass and percentage of body fat were noted. There was a concomitant higher fat free mass in the rugby and dragon boat players when compared to the controls, whist the percentage of body fat was significantly lower for these two groups. Flexibility was also higher in the rugby and dragon boat players when compared to the control group participants. The percentage of time spent in MVPA between rugby, dragon boat and controls was analyzed using one-way ANOVA.

Conclusion: These preliminary findings provide evidence into the benefits of university sports participation and much needed evidence in the role sports such as dragon boat and rugby play in musculoskeletal strength, flexibility and the possible effect on body composition.
\end{abstract}

\section{Keywords}

Rugby; Dragon boat; Musculoskeletal strength; Body composition Chinese; College students; Grip strength

\section{Introduction}

Active participation in university team sport programs provides opportunities for physical activity and contributes to the promotion of exercise related to health benefits. Team sports are gaining popularity in Asia and nowhere this is more apparent than in China, which is experiencing economic success and rapid population

*Corresponding author: Robin Mellecker, The University of Hong Kong, Institute of Human Performance, Hong Kong Jockey Club Building for Interdisciplinary Research, 5 Sassoon Road, Pokfulam, Hong Kong, E-mail: rrmel@netvigator.com

Received: March 1, 2016 Accepted: April 26, 2016 Published: April 30, 2016 growth [1]. Recommendations for developing and maintaining musculoskeletal health suggest that individuals participate in exercise that increases musculoskeletal and bone strength as well as improvements in flexibility [2]. These guidelines provide welldefined recommendations for engaging in resistance training and flexibility exercises and the dose (duration and frequency) of exercise required to produce intended outcomes. Sports participation requires coordination of the musculoskeletal system to produce body movements, which translates to gains in muscle and bone strength as well as increased flexibility. These components are the foundation of the musculoskeletal system and are known to be associated healthrelated aspects of physical fitness and many health benefits [3-5].

The introduction of rugby as an Olympic sport and Rugby Union investments have increased participation rates worldwide [6]. Rugby is a physically demanding sport requiring musculoskeletal components such as strength and flexibility [7]. Ball handling skills fundamental to playing rugby improve upper body strength [8] and a lack of flexibility decreases sports performance and increases risk of injury [9]. Despite the importance of upper body strength and flexibility and the documented risk associated with musculoskeletal injuries [10] few studies have concentrated on these components of the musculoskeletal system [11].

Traditional forms of exercise that connect individuals with cultural traditions have invigorated an interest in sports that are culturally relevant. In Asia, the need to continue with cultural specific traditions has increased interest in sports such as dragon boat. Dragon boat is a competitive water sport that requires rowers to paddle to the rapid beat of a drum over $200 \mathrm{~m}$ to $2000 \mathrm{~m}$ distances. The paddling stroke involves gripping and repetitive trunk flexion and back extension. Rowers performing these repetitive motions report a high incidence of lower back pain $[12,13]$. Improving hamstring flexibility is recommended to lessen the pain and improve sports performance [14].

Maintaining optimal levels of body composition is an important attribute for sports participation specifically for those sports that require a combination of muscle strength, aerobic and anaerobic metabolism. For contact sports such as rugby a higher percentage of body fat is desirable for tackling and collisions but may also negatively affect performance since excess body fat increases metabolic demand [15].

Studies investigating the musculoskeletal strength between amateur university sports team participants and university students who do not participate in regular organized sport in the Chinese population are nonexistent. The main purpose of this study was to compare the musculoskeletal strength and flexibility between university sports team participants and control participants. Our primary hypotheses were that: musculoskeletal strength and flexibility will be greater in rugby teams and dragon boat participants than in control participants. As our secondary aim was to examine differences in body composition and habitual activity between the three groups, we hypothesized that the rugby and dragon boat team will have higher fat free mass, lower body fat $\%$ and higher bone strength, plus higher habitual physical activity than the control participants. 


\section{Materials and Methods}

\section{Participants}

Sixty undergraduate male students (rugby players, $n=20$, dragon boat, $n=20$ and controls, $n=20$ ) were recruited from a local university. The inclusion criteria included males, aged 18-25 years old, participating in regular rugby or dragon boat training. Participants were excluded from participation from this study if they reported restrictions to physical activity, cardiopulmonary or metabolic diseases and/or musculoskeletal injuries that affect body movement. Participants in the control group followed the same inclusion and exclusion criteria mentioned above except that they did not participate in rugby or dragon boat training. The University Institutional Board of Ethics approved this study Written informed consent was obtained from each participant prior to data collection and all components of the study were conducted in compliance with sport and exercise science ethical standards [16].

\section{Procedures}

This study used a cross-sectional experimental design to assess musculoskeletal strength and body composition in dragon boat, rugby team players and controls. All participants were asked to arrive at the laboratory for a one-hour testing session including a battery of musculoskeletal strength assessments. All laboratory assessments were performed in the afternoon to account for diurnal variations in strength outcomes [17]. Following the physical tests, participants were asked to complete a short sports participation questionnaire and given the GT3X+ accelerometer (with wear instructions) to assess their habitual physical activity levels.

\section{Anthropometric assessments}

Anthropometric data were measured including body weight, height, waist and hip circumference and body composition. Stature and body composition was measured barefoot using a stadiometer (SECA 217, Chino, CA) with an accuracy of $0.1 \mathrm{~cm}$. Bioelectrical impedance (Tanita Body Composition Analyzer, TBF-410GS, IL, USA) was used to determine fat fee mass (FFM) and percentage (\%) of body fat (BF\%).

\section{Sit and reach test}

The sit and reach test was used to measure lower back and hamstring flexibility. The participants were asked to sit barefoot with their legs extended and the bottom of their feet pressed firmly against the sit-and-reach box. Once the participant was in the correct seated position, both hands were placed flat on top of each other and their fingers fully extended. The participants were instructed to slowly reach as far forward as possible without any pain to their hamstring or lower back. The furthest point in centimeters reached with the hands remaining in contact was recorded. The participants performed three trials and the average of the three scores was recorded for further analysis.

\section{Hand grip strength}

We used hand grip assessments in this study due to the importance of the forearm flexor musculature for sport performance as well as active daily living $[18,19]$ and to ensure the safety of our participants, which may not be able to perform highly skilled 1RM tests. The protocol for hang-grip strength was in accordance with previous research and recommendations on assessment to enable comparison across studies [20]. Maximal grip strength of both hands was assessed using a Jamar Analogue Hand Dynamometer (Sammons Preston, ON, Canada). A trained research assistant demonstrated the protocol seated with the elbow placed by the side of the body and flexed to $90^{\circ}$ and the wrist extended in neutral position. The participants were given the opportunity to ask questions about the protocol and when comfortable they were asked to follow the instructions as shown and squeeze the dynamometer with as much force as possible being careful to squeeze only once for each measurement. Three trails with one-minute rest periods were performed with the dominant hand and averaged for analysis [21].

\section{Bone strength of distal radius}

An Ultrasound Bone Sonometer (Sunlight BeamMed Ltd, Israel) was used to assess the bone strength of the right distal radius (see description of procedures) [22]. This system was found to be very reliable with precision of $0.36 \%$ measured at the distal radius and precise in vivo $(0.4 \%-0.8 \%)$ [23]. The Speed of Sound (SOS) value, T-score and a Z- score refer to the units of standard deviations (SD) relative to the population reference values of ethnicity-matched healthy young adults and ethnicity-age, and sex-matched population, respectively.

\section{Physical activity monitoring}

Participants completed a short sports participation questionnaire including the following questions to determine the participants' interest and competitive level of sports participation, sports of interest and the frequency and duration of sports participation: 1) Which category best describes your involvement in sport?, 2) Which sports do you participate in?, and 3) How many hours do you participate in sports per week?

In addition, participants were fitted with a GT3X+ accelerometer on their waist and instructed to wear the monitor for four days (3 weekdays and 1 weekend day). The GT3X+ activity monitor was initialized in tri-axial mode, set to record at $30 \mathrm{~Hz}$ and 60 -second epoch length.

\section{Statistical analysis}

Means and standard deviations were computed for all key variables. Normality of data was assessed using Shapiro-Wilk tests. One-way analysis of variance (ANOVA) was used to compare the demographic characteristics among the three groups. Statistically significant between-group differences in any of the demographic variables were entered into one-way analysis of covariance (ANCOVA) model as covariates. A one-way ANCOVA model was designed with independent variables bone strength, muscle strength, flexibility and body composition scores and dependent variables participation in rugby and dragon boat and the control group to determine the impact of on the musculoskeletal outcomes. Paired samples t-tests indicated no significant differences between the amount of time or the percentage of time spent in daily moderate to vigorous physical activity (MVPA) for weekday and weekend day $(p$ 's $>0.05)$. These variables were subsequently combined for all four days of assessment and differences between rugby, dragon boat and controls were analyzed using one-way ANOVA. Significant results were further analyzed with post hoc Bonferroni multiple comparisons. The significance level was set at 0.05 (two-tailed). All statistical analysis was performed using IBM SPSS 20.0 software (IBM, Armonk, NY). 


\section{Results}

Demographic characteristics as well as sports participation of the participants are presented in Table 1 . Of the 60 participants, $88 \%$ were right handed and $12 \%$ were left-handed.

Results revealed that there was a significant difference in body weight among the three groups ( $\mathrm{p}=0.031$ ). In subsequent ANCOVA analysis this variable was treated as a covariate.

Radial bone strength was comparable among the three groups $(p>0.05)$. However, significant between-group differences in handgrip strength right hand $(p=0.012)$ and left hand $(p<0.001)$, flexibility $(p<0.001)$, fat free mass $(p<0.001)$ and percentage of body fat $(p<0.001)$ were noted. Specifically, rugby $(p=0.007$ right hand; $p<0.001$ left hand) and dragon boat players $(p<0.001$ right and left hand) had significantly higher handgrip strength than the control participants. There was a concomitant higher fat free mass in the rugby $(p<0.001)$ and dragon boat players $(p<0.001)$ when compared to the controls, whist the percentage of body fat was significantly lower in these two groups of participants (rugby players vs. controls, $p<0.001$; dragon boat players vs. controls, $p=0.008$ ). Flexibility was also higher in the rugby $(p=0.001)$ and dragon boat players $(p<0.001)$ when compared to the control group participants (Table 2).

Rugby and dragon boat players spent more time playing organized sport than controls. The control group reported spending the least amount of time playing organized sport. Of the 20 control participants, six reported that they spent a minimum of one hour and none of the controls reported spending more than 5 hours a week in organized sports.

More of the rugby $14(70 \%)$ and dragon boat players $15(75 \%)$ reported that they play sport at a competitive level whereas $2(10 \%)$ control participants reported competitive participation in sport-
Recreational participation in organized sports was reported to be the highest among the control participants 15 (75\%) followed by rugby $6(30 \%)$ and dragon boat players $5(30 \%)$. All of the sports team participants reported playing sport but three of the control participants reported playing no organized sport at all.

Thirty-nine of the 60 participants completed four 10-hour days of objective physical activity assessment. The rugby players and dragon boat paddlers spent more time in MVPA than the control participants (see Table 3). One-way ANOVA results showed significant differences in the overall time $(F(2,36)=4.799, p=0.014)$ as well as percentage of time spent in MVPA $(F(2,36)=4.652, p=0.016)$. Follow up post hoc analysis indicated significant differences in the overall time spent in MVPA $(p=0.011)$ and the percentage of time in MVPA $(p=0.013)$ between the rugby players and controls. No significant differences in MVPA between the dragon boat paddlers and controls were notable $(p>0.05)$.

\section{Discussion}

These findings provide useful information on the associated benefits of participating in amateur university rugby and dragon boat sport teams. As expected, rugby and dragon boat players had significantly higher musculoskeletal strength than the control participants. This was highlighted by the significant differences in hand grip strength between groups. Hand grip strength has been used to identify potential sporting talents, injury prevention, detect overall body strength and physical condition as well as participation in daily physical activity $[18,19,24]$. The differences in hand grip strength between the sports teams and controls are not surprising given that both dragon boat and rugby require upper body strength for paddling and ball handling, respectively $[8,15]$. The control participants in our study reported involvement in recreational sport but limited participation in competitive sports and consequently lower grip

Table 1: Descriptive characteristics for the rugby, dragon boat and control participants.

\begin{tabular}{|l|c|c|c|c|c|}
\hline & Rugby players $\mathbf{( n = 2 0 )}$ & Dragon boat paddlers $\mathbf{( n = 2 0 )}$ & Controls $\mathbf{( n = 2 0 )}$ & Overall $(\mathbf{n = 6 0 )}$ & $\mathbf{P}$ value \\
\hline Age, years & $21 \pm 1$ & $21 \pm 1$ & $20 \pm 2$ & 0.441 \\
\hline Height, $\mathrm{cm}$ & $172.3 \pm 4.8$ & $172.9 \pm 4.1$ & $171.8 \pm 6.7$ & 0.797 \\
\hline Weight, kg & $68.6 \pm 7.2$ & $69.6 \pm 6.3$ & $62.7 \pm 11.5$ & $67.0 \pm 9.0$ \\
\hline Waist-hip ratio & $0.80 \pm 0.04$ & $0.82 \pm 0.04$ & $0.80 \pm 0.04$ & $0.81 \pm 0.04$ \\
\hline
\end{tabular}

${ }^{*} P<0.05$

Table 2: Body composition variables among the three groups.

\begin{tabular}{|c|c|c|c|c|c|c|}
\hline & Rugby players $(n=20)$ & Dragon boat players $(n=20)$ & Controls $(n=20)$ & All $(n=60)$ & $P$ value & F value \\
\hline \multicolumn{7}{|c|}{ Bone strength (right distal radius) } \\
\hline T score & $-1.48 \pm 0.79$ & $-1.42 \pm 0.95$ & $-1.58 \pm 1.10$ & $-1.493 \pm 0.941$ & 0.848 & 0.166 \\
\hline Z score & $-0.21 \pm 0.71$ & $-0.15 \pm 0.90$ & $-0.18 \pm 0.99$ & $-0.18 \pm 0.85$ & 0.960 & 0.041 \\
\hline \multicolumn{7}{|c|}{ Muscle strength } \\
\hline Handgrip strength (right, $\mathrm{kg}$ ) & $45.75 \pm 8.12^{\mathrm{a}}$ & $47.90 \pm 7.07^{b}$ & $37.68 \pm 8.75$ & $43.78 \pm 9.04$ & $0.012^{*}$ & 4.769 \\
\hline $\begin{array}{l}\text { Handgrip strength } \\
\text { (left, kg) }\end{array}$ & $42.73 \pm 6.62^{\mathrm{a}}$ & $44.54 \pm 6.10^{b}$ & $34.20 \pm 7.76$ & $40.50 \pm 8.13$ & $0.000^{*}$ & 12.954 \\
\hline \multicolumn{7}{|c|}{ Flexibility } \\
\hline Sit-and-reach test (cm) & $21.33 \pm 6.21^{\mathrm{a}}$ & $22.12 \pm 6.16^{b}$ & $13.22 \pm 7.06$ & $18.88 \pm 7.56$ & $<0.00^{*}$ & 9.291 \\
\hline \multicolumn{7}{|l|}{ Body composition } \\
\hline Fat free mass $(\mathrm{kg})$ & $60.87 \pm 4.98^{a}$ & $60.21 \pm 4.56^{b}$ & $51.15 \pm 6.97$ & $57.41 \pm 7.09$ & $<0.00^{*}$ & 29.39 \\
\hline Body fat $\%$ & $10.99 \pm 2.96^{a}$ & $13.19 \pm 6.09^{b}$ & $17.81 \pm 4.43$ & $13.99 \pm 5.4$ & $<0.00^{\star}$ & 31.529 \\
\hline
\end{tabular}

Values are presented as mean \pm SD.

${ }^{*} p<0.05$

aSignificant difference between rugby players

and controls $(p<0.05)$

bSignificant difference between dragon boat

players and controls $(p<0.05)$. 
Table 3: Daily physical activity levels for all three groups.

\begin{tabular}{|l|l|l|l|l|}
\hline & Rugby players $(\mathbf{n}=\mathbf{1 5})$ & Dragon boat players $(\mathbf{n}=\mathbf{1 3})$ & Controls $(\mathbf{n}=\mathbf{1 1})$ & Overall $(\mathbf{n}=\mathbf{3 9})$ \\
\hline MVPA minutes/day & $85.47 \pm 30.08^{*}$ & $73.76 \pm 25.30$ & $54.18 \pm 17.39^{*}$ & $72.74 \pm 27.91$ \\
\hline \% Time spent MVPA & $6.3 \pm 2.2^{*}$ & $5.3 \pm 1.9$ & $4.0 \pm 1.3^{*}$ & $0.014^{*}$ \\
\hline
\end{tabular}

${ }^{*} p<0.05$

strength than the sport team players. This is concurrent with previous research, which suggests a positive relationship between sports participation and hand grip strength [18]. Surprisingly, the overall average values for hand grip strength are well below the international normative data for the age and gender of the participants in this study [25]. Given the possible implications and reported association between hand grip strength and long term health risk, [19] there appears to be a need for intervention in this population [26].

This study provides support for the recent recommendation that flexibility exercises should be performed at least twice a week to maintain functional status [27]. Flexibility is crucial in preventing sports injuries [28] as well as reducing lower back pain [29]. In our present study, sit and reach test was used to measure the flexibility of hamstring. According to previous studies, limited range of motion in the lower limbs is shown to increase sport injuries [10]. Hamstring flexibility greatly improves performance by reducing muscle stiffness and increasing the range of motion to cater for the demand of the muscular contraction and movement required in sports [14]. For the control group, hamstring flexibility may greatly reduce the chance of lower back pain, because tightness in the hamstring increases the pressure around the lower lumbar region, as a result increasing the risk for lower back injuries [25].

We examined the body composition characteristics of our participants and found differences in fat free mass and BF\% between sports team members and control participants. Studies documenting the body composition characteristics of dragon boat paddlers and rugby players suggest variance in body fat and fat free mass [31,32], which may be explained by differences in training regimes, level of professionalism (amateur and elite) [33] and changes in body size of athletes over time $[32,34]$. Overall our participants have a lower BF\% and higher fat free mass than Asian college aged students and lower BF\% and fat free mass than other Asian ethnic groups living in the US [35].

We found no differences in bone strength despite the strong relationship with weight bearing exercise commensurate with sports such as rugby. The participants in this study were all healthy young adults and we found no differences in lean mass, which is a predictor of bone health [36]. Our findings are contrary to those reported elsewhere, which have reported higher BMC in amateur and elite athletes [36,37]. These differences are noteworthy and deserve further exploration given the projected increase in osteoporosis in Asia [38].

The control participants report spending the least amount of time playing organized sport when compared to rugby and dragon boat players. Sports participation levels and the time spent playing various sports may explain some of the differences in musculoskeletal outcomes among the sports team players and control in this study. Self-reported levels of physical activity of college students in Hong Kong indicate that this population is active [39]. The rugby team members spent a higher percentage of time in daily moderate to vigorous physical activity when compared with controls. All of the participants in this study spend more time in MVPA than collegeaged students' elsewhere [40]. Whilst these results are promising it is worth continuing to follow appeals for the promotion of university sports programs given the lack of upper body strength and flexibility noted in this study [41].

This study focused on Hong Kong Chinese college students, which limits the generalization of our results. However, these results add to the limited research on sports participation in Chinese college aged students, which are reported to be less active than counterparts in Western countries [42]. We used an accelerometer to assess daily physical activity levels but due to compliance we were only able to report MVPA for 39 of our 60 participants. We used a short questionnaire to assess sports participation, which did not provide intensity of training or level of participation for both sports. Comprehensive triangulated assessments would confirm these preliminary findings and add further insight into the patterns of sports participation among college-aged Asian students.

\section{Conclusions}

These preliminary findings provide evidence into the benefits of university sports participation and much needed evidence in the role sports such as dragon boat and rugby play in musculoskeletal strength, flexibility and the possible effect on body composition. This study provides preliminary evidence that university sport programs such as rugby or dragon boat may provide a gateway effect in promoting physical activity alternatives that translate into musculoskeletal and body composition improvements in Hong Kong college students. Further research to determine promotional strategies to increase participation in university team sports is warranted in this population.

\section{References}

1. Hajkowicz SA, Cook H, Littleboy A (2012) Our future world: Global megatrends that will change the way we live. The 2012 Revision. Queensland, Australia.

2. Garber CE, Blissmer B, Deschenes MR, Franklin BA, Lamonte MJ, et al. (2011) American college of sports medicine position stands. quantity and quality of exercise for developing and maintaining cardiorespiratory, musculoskeletal, and neuromotor fitness in apparently healthy adults: guidance for prescribing exercise. Med Sci Sports Exerc 43: 1334-1359.

3. Brill PA, Macera CA, Davis DR, Blair SN, Gordon N (2000) Muscular strength and physical function. Med Sci Sports Exerc 32: 412-416.

4. Kell T, Bell G, Quinney A (2001) Musculoskeletal fitness, health outcomes and quality of life. Sports Med 31: 863-873.

5. Warburton DE, Gledhill N, Quinney A (2001) The effects of changes in musculoskeletal fitness on health. Can J Appl Physiol 26: 161-216.

6. Chadwick S, Semens A, Arthur D (2011) Economic Impact on Global Rugby Part IV: Rugby World Cup 2011. Centre for the international business of sport (CIBS) Coventry, UK.

7. Gabbett TJ Science of rugby league football: A review (2005) J Sports Sci 23: $961-976$.

8. Martin-Martin J, Cuesta-Vargas A (2014) Quantification of functional hand grip using electromyography and inertial sensor-derived accelerations: clinical implications. BioMed Eng OnLine 13: 161.

9. Brooks J, Fuller C, Kemp S, Reddin D (2006) Incidence, risk, and prevention of hamstring muscle injuries in professional rugby union. Am J Sports Med 34: 1297-1306.

10. Brooks J, Kemp S (2008) Recent trends in rugby union injuries. Clin Sports Med 27: 51-73 
Citation: Mellecker RR, Fong SSM, Macfarlane DJ, Zhang J, Wu KM (2016) Comparison of Musculoskeletal Strength and Body Composition of Hong Kong Chinese Rugby Players, Dragon Boat Paddlers and Controls. J Athl Enhancement 5:2.

11. Fletcher IM, Jones B (2004) The effect of different warm-up stretch protocols on 20 -meter sprint performance in trained rugby union players. J Strength Cond Res 18: 885-888.

12. Newlands $C$, Reid D, Parma $P(2015)$ The prevalence, incidence and severity of low back pain among international-level rowers. Br J Sports Med 49: 951-956.

13. Teitz CC, O'Kane J, Lind BK, Hannafin JA (2002) Back pain in intercollegiate rowers. Am J Sports Med 30: 674-679.

14. Shrier I (2004) Does stretching improve performance?: A systematic and critical review of the literature. Clin J Sport Med 14: 267-273.

15. Meir R, Newton R, Curtis E, Fardell M, Butler B (2001) Physical fitness qualities of professional rugby league players: Determination of positional differences. J Strength Cond 15: 450-458.

16. Harris DJ, Atkinson G (2013) Ethical standards in sport and exercise science research: 2014 update. Int J Sports Med 34: 1025-1028.

17. Cappaert T (1999) Review: Time of day effect on athletic performance: an update. J Strength Cond Res 13: 412-421.

18. Aadahl M, Beyer N, Linneberg A, Betina HT, Jørgensen T (2011) Grip strength and lower limb extension power in 19-72-year-old Danish men and women The Health 2006 study. BMJ Open 2: e000192.

19. Leong DP, Teo KK, Rangarajan S, Lopez-Jaramillo P, Avezum A, et al (2015) Prognostic value of grip strength: findings from the Prospective Urban Rural Epidemiology (PURE) study. Lancet 386: 266-273.

20. Massy-Westropp NM, Gill TK, Taylor AW, Bohannon RW, Hill CL (2011) Hand grip strength: age and gender stratified normative data in a population based study. BMC Res Notes 4: 127.

21. Fong SSM, Guo X, Cheung APM, Jo ATL, Lui GKW, et al. (2013) Elder Chinese martial art practitioners has higher radial bone strength, handgrip strength and better standing balance control. Rehabil 2013

22. Knapp KM, Blake KM, Spector TD, Fogelman I (2001) Multisite quantitative ultrasound: precision, age- and menopause related changes, fracture discrimination, and T-score equivalence with dual-energy X-ray absorptiometry. Osteoporos Int 12: 456-464.

23. Barkmann R, Kantorovich E, Singal C, Hans D, Genant HK, et al. (2000) A new method for quantitative ultrasound measurements at multiple skeletal sites: First results of precision and fracture discrimination. J Clin Densitom 3: $1-7$.

24. Sirajudeen MS, Shah UN, Pillai PS, Mohasin N, Shantaram M (2012) Correlation between grip strength and physical factors in men. Int $\mathrm{J}$ Health Rehabil Sci 1: 58-63.

25. Bohannon RW, Peolsson A, Massy-Westropp N, Desrosiers J, Bear-Lehman JB (2006) Reference values for adult grip strength measured with a Jamar dynamometer: a descriptive meta-analysis. Physiotherapy 92: 11-5.

26. Bohannon RW (2008) Hand-grip dynamometry predicts future outcomes in aging adults. J Geriatr Phys Ther 31: 3-10.

27. Blair SN, LaMonte MJ, Nichaman MZ (2004) The evolution of physical activity recommendations: How much is enough? Am J Clinl Nutr. 79: 913S-920S.

28. Odunaiya NA, Hamzat TK, Ajayi OF (2005) The effects of static stretch duration on the flexibility of hamstring muscles. AJBR 8: 79-82.

29. Ylinen J, Kankainen $T$, Kautiainen $H$, Rezasoltani $A$, Kuukkanen $T$, et al. (2009) Effect of stretching on hamstring muscle compliance. J Rehabil Med 41: $80-84$.

30. Rumball JS, Lebrun CM, Di Ciacca SR, Orlando K (2005) Rowing injuries. Sports Med 35: 537-55

31. Marrin K, Pout MJ (2005) Anthropometric and physiological characteristics of elite male dragon boat paddlers. J Sports Sci 23: 1204.

32. Singh R, Singh HJ, Sirisinghe RG (1995) Physical and physiological profiles of Malaysian dragon boat rowers. Br J Sports Med 29: 13-15.

33. Smart DJ, Hopkins WG, Gill ND (2013) Differences and changes in the physical characteristics of professional and amateur rugby union players. $J$ Strength Cond Res 27: 3033-3044.

34. Olds T (2001) The evolution of physique in male rugby union players in the twentieth century. J Sports Sci 19: 253-262.
35. Carpenter CL, Yen E, Chen S, Hong K, Arechiga A, et al. (2013) Body Fat and Body-Mass Index among a Multiethnic Sample of College-Age Men and Women. J Obes.

36. Nevill AM, Holder RL, Stewart AD (2003) Modeling elite male athletes' peripheral bone mass, assessed using regional dual x-ray absorptiometry. Bone 32: 62-68.

37. Morel J, Combe B, Francisco J, Bernard J (2001) Bone mineral density of 704 amateur sportsmen involved in different physical activities. Osteoporos Int 12: 152-157.

38. Dhanwal DK, Cooper C, Dennison EM (2010) Geographic Variation in Osteoporotic Hip Fracture Incidence: The Growing Importance of Asian Influences in Coming Decades. J Osteoporos.

39. Abdullah ASM, Wong CM, Yam HK, Fielding R (2005) Factors related to non-participation in physical activity among the students in Hong Kong. Int $J$ Sports Med 26: 611-615.

40. Downs A, Van Hoomissen J, Lafrenz A, Julka DL (2014). Accelerometermeasured versus self-reported physical activity in college students: implications for research and practice. J Am Coll Health 62: 204-212.

41. Center for Disease Control and Prevention (2008) Physical Activity Guidelines for Americans. Atlanta, GA: U. S. Department of Health and Human Services, Office of Disease Prevention and Health Promotion.

42. Bauman A, Bull F, Chey T, Craig LC, Ainsworth B, et al. (2009) The international prevalence study on physical activity: results from 20 countries. Int J Behav Nutr Phys Act 6: 21.

\section{Author Affiliation}

${ }^{1}$ University of Hong Kong, Institute of Human Performance, Hong Kong Jockey Club for Interdisciplinary Research Building, 5 Sassoon Road, Pokfulam, Hong Kong

${ }^{2}$ Centre for Physical Activity \& Nutrition Research (C-PAN), School of Exercise and Nutrition Sciences, Faculty of Health, Deakin University, Melbourne, Australia

${ }^{3}$ Department of Sports Science and Physical Education, The Chinese University of Hong Kong

Submit your next manuscript and get advantages of SciTechnol submissions
* 50 Journals
* 21 Day rapid review process
* 1000 Editorial team
* 2 Million readers
* Publication immediately after acceptance
* Quality and quick editorial, review processing

Submit your next manuscript at $\bullet$ www.scitechnol.com/submission 